CRYSTALLOGRAPHIC COMMUNICATIONS

ISSN 2056-9890

Received 1 December 2015

Accepted 15 January 2016

Edited by P. Roussel, ENSCL, France

Keywords: crystal structure; apatite structure type; calcium rare earth oxide silicate

CCDC reference: 1447637

Supporting information: this article has supporting information at journals.iucr.org/e

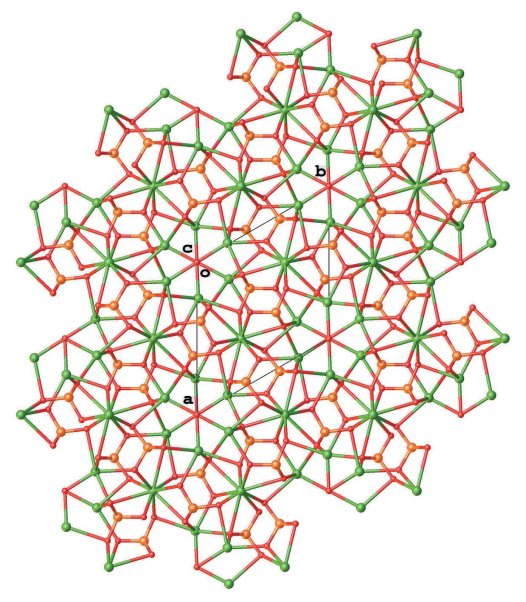

OPEN $\odot$ ACCESS

\section{Crystal structure of apatite type $\mathrm{Ca}_{2.49} \mathrm{Nd}_{7.51}\left(\mathrm{SiO}_{4}\right)_{6} \mathrm{O}_{1.75}$}

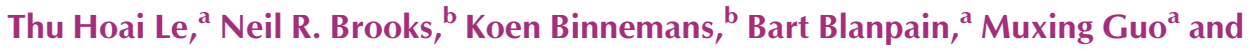 \\ Luc Van Meervelt ${ }^{b_{*}}$ *
}

${ }^{a} \mathrm{KU}$ Leuven - University of Leuven, Department of Metallurgy and Materials Engineering, Kasteelpark Arenberg 44 - bus
2450, B-3001 Heverlee, Belgium, and ${ }^{\mathbf{b}} \mathrm{KU}$ Leuven - University of Leuven, Department of Chemistry, Celestijnenlaan
200F - bus 2404, B-3001 Heverlee, Belgium. *Correspondence e-mail: luc.vanmeervelt@chem.kuleuven.be

The title compound, $\mathrm{Ca}_{2+x} \mathrm{Nd}_{8-x}\left(\mathrm{SiO}_{4}\right)_{6} \mathrm{O}_{2-0.5 x}(x=0.49)$, was synthesized at $1873 \mathrm{~K}$ and rapidly quenched to room temperature. Its structure has been determined using single-crystal X-ray diffraction and compared with results reported using neutron and X-ray powder diffraction from samples prepared by slow cooling. The single-crystal structure from room temperature data was found to belong to the space group $P 6_{3} / m$ and has the composition $\mathrm{Ca}_{2.49} \mathrm{Nd}_{7.51}\left(\mathrm{SiO}_{4}\right)_{6} \mathrm{O}_{1.75}$ [dicalcium octaneodymium hexakis(orthosilicate) dioxide], being isotypic with natural apatite and the previously reported $\mathrm{Ca}_{2} \mathrm{Nd}_{8}\left(\mathrm{SiO}_{4}\right)_{6} \mathrm{O}_{2}$ and $\mathrm{Ca}_{2.2} \mathrm{Nd}_{7.8}\left(\mathrm{SiO}_{4}\right)_{6} \mathrm{O}_{1.9}$. The solubility limit of calcium in the equilibrium state at $1873 \mathrm{~K}$ was found to occur at a composition of $\mathrm{Ca}_{2+x} \mathrm{Nd}_{8-x}\left(\mathrm{SiO}_{4}\right)_{6} \mathrm{O}_{2-0.5 x}$, where $x=0.49$.

\section{Chemical context}

The study of calcium rare earth oxide silicates is important because they are usually observed in nuclear waste along with rare earth silicates. So far, the calcium rare earth oxide silicates of Nd (Fahey \& Weber, 1982; Fahey et al., 1985), Sm (PDF 29-365; Smith, 1977), Eu (PDF 29-320; Smith, 1977), Gd (PDF 28-212; Smith, 1976), Tb (PDF 38-256; Lacout, 1986), and Ce (Skakle et al., 2000) have been studied. Fahey \& Weber et al. (1982) and Fahey et al. (1985) published the structure and stoichiometry limits of the $\mathrm{Ca}_{2+x} \mathrm{Nd}_{8-x}\left(\mathrm{SiO}_{4}\right)_{2-0.5 x}$ system using $\mathrm{X}$-ray and neutron powder diffraction. In that study, the samples were synthesized at 1523 or $1873 \mathrm{~K}$ and cooled at a rate of $250 \mathrm{~K}$ per hour. However, such a slow cooling process may lead to undesired modifications of the obtained specimens since the solubility of calcium does not remain constant but decreases with decreasing temperature. This problem is avoided in the present work by rapid quenching of the $\mathrm{Ca}_{2+x} \mathrm{Nd}_{8-x}\left(\mathrm{SiO}_{4}\right)_{6} \mathrm{O}_{2-0.5 x}$ samples in their equilibrium state at $1873 \mathrm{~K}$ to room temperature within a few seconds. Consequently, compositions of the samples can be preserved better.

\section{Structural commentary}

The single crystal structure determined from room temperature data was found to belong to the space group $\mathrm{Pb}_{3} / \mathrm{m}$ and has the composition $\mathrm{Ca}_{2.49} \mathrm{Nd}_{7.51}\left(\mathrm{SiO}_{4}\right)_{6} \mathrm{O}_{1.75}$ and is isotypic with natural apatite and the previously reported $\mathrm{Ca}_{2} \mathrm{Nd}_{8}\left(\mathrm{SiO}_{4}\right)_{6} \mathrm{O}_{2}$ and $\mathrm{Ca}_{2.2} \mathrm{Nd}_{7.8}\left(\mathrm{SiO}_{4}\right)_{6} \mathrm{O}_{1.9}$ (Fahey \& Weber, 1982; Fahey et al., 1985). The solubility limit of calcium in the equilibrium state at $1873 \mathrm{~K}$ was found to occur at a composition of $\mathrm{Ca}_{2+x} \mathrm{Nd}_{8-x}\left(\mathrm{SiO}_{4}\right)_{6} \mathrm{O}_{2-0.5 x}$, where $x=0.49$. 


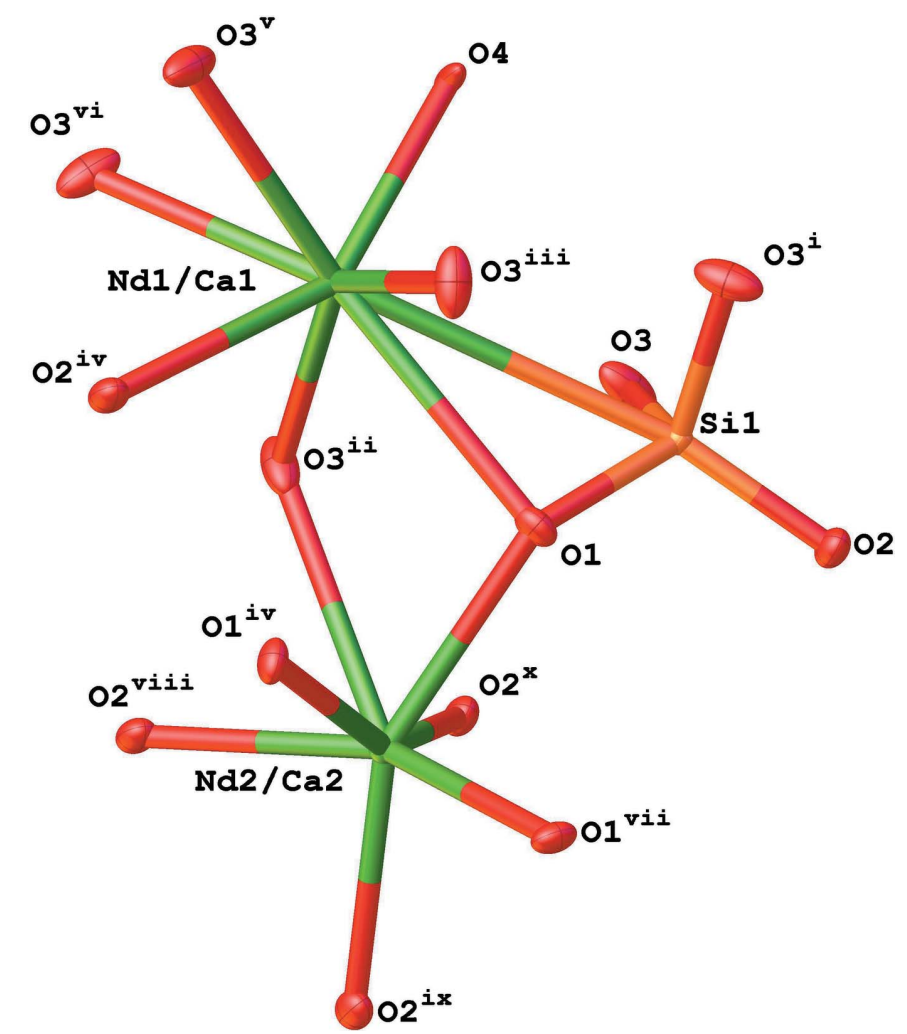

Figure 1

View of the coordination spheres of the $\mathrm{Nd} / \mathrm{Ca}$ and $\mathrm{Si}$ atoms [displacement ellipsoids shown at the $50 \%$ probability level; symmetry codes: (i) $x, y,-z+\frac{1}{2}$; (ii) $y,-x+y,-z$; (iii) $y,-x+y, z+\frac{1}{2}$; (iv) $-y+1$, $x-y, z$; (v) $y-x,-x,-z+\frac{1}{2}$; (vi) $y-x,-x, z$; (vii) $y-x+1,-x+1, z$; (viii) $y,-x+y, z-\frac{1}{2}$; (ix) $-y+x+1, x, z-\frac{1}{2}$; (x) $\left.-x+1,-y+1, z-\frac{1}{2}\right]$.

There are two metal positions in the asymmetric unit of the structure (Fig. 1) and both contain disordered $\mathrm{Nd}$ and $\mathrm{Ca}$ ions: $\mathrm{Nd} 1 / \mathrm{Ca} 1$ occupies the lower symmetry site $6 h$ and $\mathrm{Nd} 2 / \mathrm{Ca} 2$ the higher symmetry site $4 f$. The occupancies of these metal sites were refined resulting in $0.887(5) / 0.113$ (5) for $\mathrm{Nd} 1 / \mathrm{Ca} 1$ and $0.546(4) / 0.454$ (4) for $\mathrm{Nd} 2 / \mathrm{Ca} 2$. The majority $(80 \%)$ of calcium is situated at the $4 f$ site. In the structures of $\mathrm{Ca}_{2} \mathrm{Nd}_{8}\left(\mathrm{SiO}_{4}\right)_{6} \mathrm{O}_{2}$ and $\mathrm{Ca}_{2.2} \mathrm{Nd}_{7.8}\left(\mathrm{SiO}_{4}\right)_{6} \mathrm{O}_{1.9}$, these values are 89 and $73 \%$, respectively (Fahey et al., 1985). The refined value of the amount of $\mathrm{Nd}$ in the structure gives a value of 0.49 for $x$ in the equation $\mathrm{Ca}_{2+x} \mathrm{Nd}_{8-x}\left(\mathrm{SiO}_{4}\right)_{6} \mathrm{O}_{2-0.5 x}$. For charge-balance purposes, the occupancy of $\mathrm{O}^{2-}$ in the structure must be $2-0.5 x$ or 1.755 . Initially, the occupancy of the $\mathrm{O}^{2-}$ position $\mathrm{O} 4$ in the structure was allowed to refine freely and its value was close to what is required for charge balance; however, it was fixed at 0.146 as the refinement of heavy-atom positions is the most reliable and exact charge balance is required.

The $\mathrm{Nd} 1 / \mathrm{Ca} 1$ site is seven coordinate and the $\mathrm{Nd} / \mathrm{Ca}-\mathrm{O}$ bond lengths vary between 2.3909 (19) and 2.721 (3) $\AA$ for oxygen atoms of the $\mathrm{SiO}_{4}{ }^{2-}$ unit but the shortest bond length of 2.2681 (2) $\AA$ is to the $\mathrm{O}^{2-}$ ion, O4 (Fig. 1; Table 1). The $\mathrm{Nd} 2 /$ $\mathrm{Ca} 2$ site is nine coordinate and only bonds to $\mathrm{SiO}_{4}{ }^{2-}$ units with six short distances $[\mathrm{Nd}-\mathrm{O}=2.4231(17), 2.4715(18) \AA]$ and three long distances $[\mathrm{Nd}-\mathrm{O}=2.830$ (2) $\AA$ ] (Fig. 1; Table 1) are observed. The distances are similar to those reported by
Table 1

Selected bond lengths $(\AA)$.

\begin{tabular}{llll}
\hline $\mathrm{Nd} 1-\mathrm{O} 1$ & $2.721(3)$ & $\mathrm{Nd} 2-\mathrm{O} 2^{\mathrm{iv}}$ & $2.4715(18)$ \\
$\mathrm{Nd} 1-\mathrm{O} 2^{\mathrm{i}}$ & $2.463(3)$ & $\mathrm{Nd} 2-\mathrm{O} 3^{\mathrm{v}}$ & $2.830(2)$ \\
$\mathrm{Nd} 1-\mathrm{O}^{\mathrm{ii}}$ & $2.3909(19)$ & $\mathrm{Si} 1-\mathrm{O} 1$ & $1.621(3)$ \\
$\mathrm{Nd} 1-\mathrm{O} 3^{\mathrm{iii}}$ & $2.547(2)$ & $\mathrm{Si} 1-\mathrm{O} 2$ & $1.623(3)$ \\
$\mathrm{Nd} 1-\mathrm{O} 4$ & $2.2681(2)$ & $\mathrm{Si} 1-\mathrm{O} 3^{\mathrm{vi}}$ & $1.629(2)$ \\
$\mathrm{Nd} 2-\mathrm{O} 1^{\mathrm{i}}$ & $2.4231(17)$ & & \\
\hline
\end{tabular}

Symmetry codes: (i) $-y+1, x-y, z$; (ii) $y,-x+y, z+\frac{1}{2}$; (iii) $-x+y,-x,-z+\frac{1}{2}$; (iv) $x-y+1, x, z-\frac{1}{2}$; (v) $-x+1,-y+1,-z$; (vi) $x, y,-z+\frac{1}{2}$.

Fahey et al. (1985) for the structures of $\mathrm{Ca}_{2} \mathrm{Nd}_{8}\left(\mathrm{SiO}_{4}\right)_{6} \mathrm{O}_{2}$ and $\mathrm{Ca}_{2.2} \mathrm{Nd}_{7.8}\left(\mathrm{SiO}_{4}\right)_{6} \mathrm{O}_{1.9}$ determined by powder $\mathrm{X}$-ray diffraction.

The $\mathrm{O} 4$ atom $\left(\mathrm{O}^{2-}\right.$ ion $)$ is coordinated to three different $\mathrm{Nd} 1 / \mathrm{Ca} 1$ ions whilst the $\mathrm{SiO}_{4}{ }^{4-}$ group has eight contacts to different $\mathrm{Nd} / \mathrm{Ca}$ positions. The $\mathrm{O} 1$ atom coordinates one $\mathrm{Nd} 1 / \mathrm{Ca} 1$ position and two $\mathrm{Nd} 2 / \mathrm{Ca} 2$ positions, the $\mathrm{O} 2$ atom coordinates one $\mathrm{Nd} 1 / \mathrm{Ca} 1$ position and two $\mathrm{Nd} 2 / \mathrm{Ca} 2$ positions and the $\mathrm{O} 3$ position coordinates one $\mathrm{Nd} 1 / \mathrm{Ca} 1$ and one $\mathrm{Nd} 2 /$ $\mathrm{Ca} 2$ positions. These contacts generate the packing, which can be seen viewed down the $c$ axis in Fig. 2 .

\section{Synthesis and crystallization}

A mixture of appropriate amounts of fine powders of $\mathrm{Nd}_{2} \mathrm{O}_{3}$ (99.99\%), $\mathrm{CaO}(99.9 \%)$ and $\mathrm{SiO}_{2}(99.9 \%)$ was put into a sealed Pt-20\%Rh tube and heated to $1873 \mathrm{~K}$ in an argon atmosphere and maintained at that temperature for $24 \mathrm{~h}$. $\mathrm{CaO}$

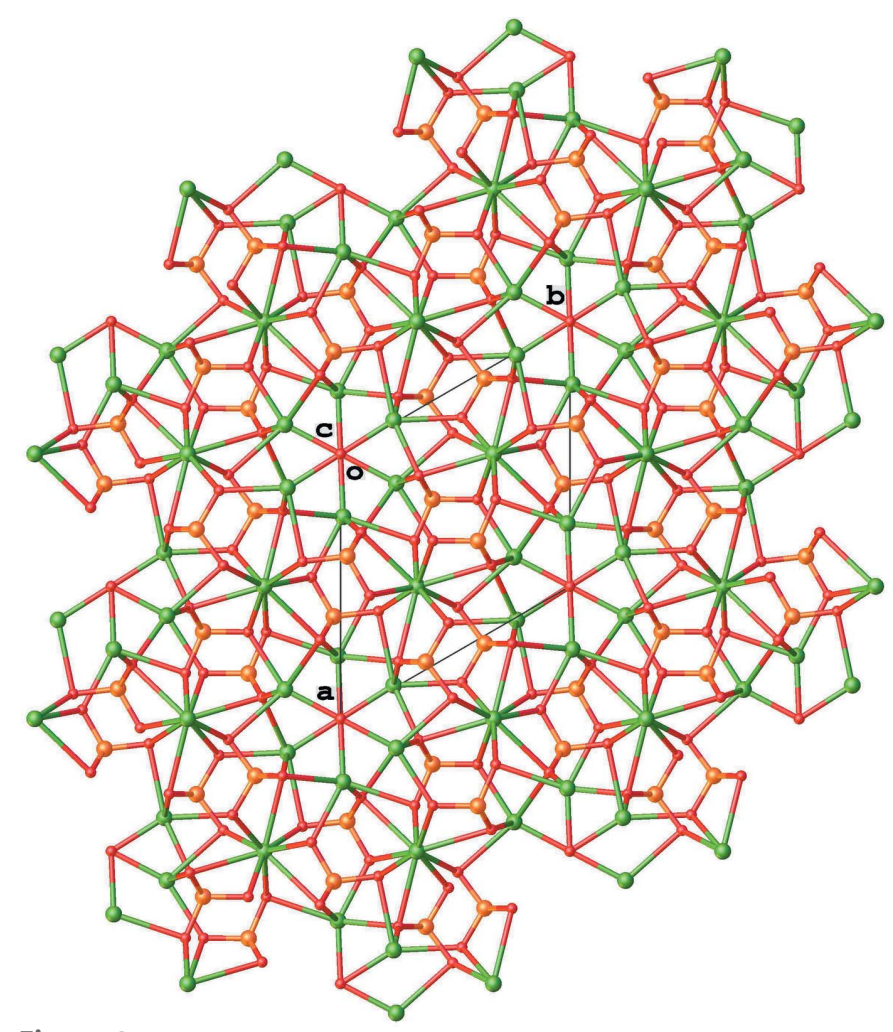

Figure 2

View along the $c$ axis of the packing arrangement. 
Table 2

Experimental details.

\begin{tabular}{ll}
\hline Crystal data & \\
Chemical formula & $\mathrm{Ca}_{2.49} \mathrm{Nd}_{7.51}\left(\mathrm{SiO}_{4}\right)_{6} \mathrm{O}_{1.75}$ \\
$M_{\mathrm{r}}$ & 1763.24 \\
Crystal system, space group & Hexagonal, $P 6_{3} / m$ \\
Temperature $(\mathrm{K})$ & 298 \\
$a, c(\AA)$ & $9.5507(3), 7.0513(3)$ \\
$V\left(\AA^{3}\right)$ & $557.03(3)$ \\
$Z$ & 1 \\
Radiation type & Mo $K \alpha$ \\
$\mu\left(\mathrm{mm}^{-1}\right)$ & 18.18 \\
Crystal size (mm) & $0.05 \times 0.05 \times 0.05$ \\
& \\
Data collection & Agilent SuperNova (single source \\
Diffractometer & at offset, Eos detector) \\
& Multi-scan $(C r y s A l i s ~ P R O ;$ \\
Absorption correction & Agilent, 2012) \\
& $0.717,1.000$ \\
$T_{\min }, T_{\max }$ & $2616,878,813$ \\
No. of measured, independent and & \\
$\quad$ observed $[I>2 \sigma(I)]$ reflections & 0.024 \\
$R_{\text {int }}$ & 0.821 \\
$(\text { sin } \theta / \lambda)_{\max }\left(\AA^{-1}\right)$ & \\
Refinement & \\
$R\left[F^{2}>2 \sigma\left(F^{2}\right)\right], w R\left(F^{2}\right), S$ & $0.019,0.035,1.11$ \\
No. of reflections & 878 \\
No. of parameters & 42 \\
$\Delta \rho_{\text {max }}, \Delta \rho_{\text {min }}(\mathrm{e} \AA$ & \\
\hline
\end{tabular}

Computer programs: CrysAlis PRO (Agilent, 2012), SHELXS97 (Sheldrick, 2008), SHELXL2014 (Sheldrick, 2015), OLEX2 (Dolomanov et al., 2009) and publCIF (Westrip, 2010).

was made by calcination of $\mathrm{CaCO}_{3}$ at $1373 \mathrm{~K}$ for $12 \mathrm{~h}$. The sample was then quenched in a cold-water bath to give a lightblue crystalline solid, from which a single crystal of the title compound was selected. The sample was further analyzed by EPMA-WDS, giving a composition of $20.2 \% \mathrm{SiO}_{2}, 72.1 \%$ $\mathrm{Nd}_{2} \mathrm{O}_{3}$ and $7.7 \% \mathrm{CaO}$. The converted formula according to the EPMA-WDS result was $\mathrm{Ca}_{2.45} \mathrm{Nd}_{7.45} \mathrm{Si}_{6} \mathrm{O}_{25.775}$ (O was calculated).

\section{Refinement details}

Crystal data, data collection and structure refinement details are summarized in Table 2. There are two metal positions in the structure and the $\mathrm{Nd}$ and $\mathrm{Ca}$ ions are disordered on both of these sites. $\mathrm{Nd} / \mathrm{Ca}$ occupancy on each of the two positions was refined and the occupancy of $\mathrm{Nd}$ was found to be 88.7 (5)\% for one site and 54.6 (4)\% for the other, giving a value of 0.49 for $x$ in $\mathrm{Ca}_{2+\mathrm{x}} \mathrm{Nd}_{8-x}\left(\mathrm{SiO}_{4}\right)_{6} \mathrm{O}_{2-0.5 x}$. The occupancy of the anionic $\mathrm{O}$ atom was fixed at $2-0.5 x$. Constraints were applied so that the $\mathrm{Nd}$ and $\mathrm{Ca}$ on the same site had identical positional and displacement parameters.

\section{Acknowledgements}

The authors thank the Hercules Foundation for supporting the purchase of the diffractometer through project AKUL/09/ 0035 .

\section{References}

Agilent (2012). CrysAlis PRO. Agilent Technologies, Yarnton, England.

Dolomanov, O. V., Bourhis, L. J., Gildea, R. J., Howard, J. A. K. \& Puschmann, H. (2009). J. Appl. Cryst. 42, 339-341.

Fahey, J. A. \& Weber, W. J. (1982). The Rare Earths in Modern Science and Technology, Vol. 3, edited by G. J. McCarthy, H. B. Silber \& J. J. Rhyne pp. 341-344. Berlin: Springer.

Fahey, J. A., Weber, W. J. \& Rotella, F. J. (1985). J. Solid State Chem. 60, 145-158.

Lacout, J. (1986). Private communication to the ICDD. PDF 38-256.

Sheldrick, G. M. (2008). Acta Cryst. A64, 112-122.

Sheldrick, G. M. (2015). Acta Cryst. C71, 3-8.

Skakle, J. M. S., Dickson, C. L. \& Glasser, F. P. (2000). Powder Diffr. 15, 234-238.

Smith, C. (1976). ICDD Grant-in-Aid. PDF 28-212.

Smith, C. (1977). ICDD Grant-in-Aid. PDF 29-365, PDF 29-320.

Westrip, S. P. (2010). J. Appl. Cryst. 43, 920-925. 


\section{supporting information}

Acta Cryst. (2016). E72, 209-211 [doi:10.1107/S205698901600089X]

Crystal structure of apatite type $\mathrm{Ca}_{2.49} \mathrm{Ndd}_{7.51}\left(\mathrm{SiO}_{4}\right)_{6} \mathrm{O}_{1.75}$

Thu Hoai Le, Neil R. Brooks, Koen Binnemans, Bart Blanpain, Muxing Guo and Luc Van Meervelt

Computing details

Data collection: CrysAlis PRO (Agilent, 2012); cell refinement: CrysAlis PRO (Agilent, 2012); data reduction: CrysAlis PRO (Agilent, 2012); program(s) used to solve structure: SHELXS97 (Sheldrick, 2008); program(s) used to refine structure: SHELXL2014 (Sheldrick, 2015); molecular graphics: OLEX2 (Dolomanov et al., 2009); software used to prepare material for publication: OLEX2 (Dolomanov et al., 2009) and publCIF (Westrip, 2010).

Dicalcium octaneodymium hexakis(orthosilicate) dioxide

Crystal data

$\mathrm{Ca}_{2.49} \mathrm{Nd}_{7.51}\left(\mathrm{SiO}_{4}\right)_{6} \mathrm{O}_{1.75}$

$M_{r}=1763.24$

Hexagonal, $P 6_{3} / m$

Hall symbol: -P 6c

$a=9.5507(3) \AA$

$c=7.0513(3) \AA$

$V=557.03(3) \AA^{3}$

$Z=1$

$F(000)=790$

\section{Data collection}

Agilent SuperNova (single source at offset, Eos detector) diffractometer

Radiation source: SuperNova (Mo) X-ray Source

Mirror monochromator

Detector resolution: 15.9631 pixels $\mathrm{mm}^{-1}$

$\omega$ scans

Absorption correction: multi-scan

(CrysAlis PRO; Agilent, 2012)

Refinement

Refinement on $F^{2}$

Least-squares matrix: full

$R\left[F^{2}>2 \sigma\left(F^{2}\right)\right]=0.019$

$w R\left(F^{2}\right)=0.035$

$S=1.11$

878 reflections

42 parameters

0 restraints

4 constraints
$D_{\mathrm{x}}=5.256 \mathrm{Mg} \mathrm{m}^{-3}$

Mo $K \alpha$ radiation, $\lambda=0.71073 \AA$

Cell parameters from 1649 reflections

$\theta=4.3-35.5^{\circ}$

$\mu=18.18 \mathrm{~mm}^{-1}$

$T=298 \mathrm{~K}$

Block, light blue

$0.05 \times 0.05 \times 0.05 \mathrm{~mm}$

$T_{\min }=0.717, T_{\max }=1.000$

2616 measured reflections

878 independent reflections

813 reflections with $I>2 \sigma(I)$

$R_{\text {int }}=0.024$

$\theta_{\max }=35.7^{\circ}, \theta_{\min }=3.8^{\circ}$

$h=-15 \rightarrow 15$

$k=-15 \rightarrow 15$

$l=-11 \rightarrow 5$

Primary atom site location: structure-invariant direct methods

Secondary atom site location: difference Fourier map

$w=1 /\left[\sigma^{2}\left(F_{\mathrm{o}}^{2}\right)+(0.0072 P)^{2}+0.3232 P\right]$

where $P=\left(F_{\mathrm{o}}^{2}+2 F_{\mathrm{c}}^{2}\right) / 3$

$(\Delta / \sigma)_{\max }=0.001$

$\Delta \rho_{\max }=0.79$ e $\AA^{-3}$

$\Delta \rho_{\min }=-0.86$ e $\AA^{-3}$ 
Extinction correction: SHELXL2014 (Sheldrick, 2015), $\mathrm{Fc}^{*}=\mathrm{kFc}\left[1+0.001 \mathrm{xFc}^{2} \lambda^{3} / \sin (2 \theta)\right]^{-1 / 4}$

\section{Special details}

Geometry. All e.s.d.'s (except the e.s.d. in the dihedral angle between two 1.s. planes) are estimated using the full covariance matrix. The cell e.s.d.'s are taken into account individually in the estimation of e.s.d.'s in distances, angles and torsion angles; correlations between e.s.d.'s in cell parameters are only used when they are defined by crystal symmetry. An approximate (isotropic) treatment of cell e.s.d.'s is used for estimating e.s.d.'s involving 1.s. planes.

Refinement. Refinement of $F^{2}$ against ALL reflections. The weighted $R$-factor $w R$ and goodness of fit $S$ are based on $F^{2}$, conventional $R$-factors $R$ are based on $F$, with $F$ set to zero for negative $F^{2}$. The threshold expression of $F^{2}>\sigma\left(F^{2}\right)$ is used only for calculating $R$-factors(gt) etc. and is not relevant to the choice of reflections for refinement. $R$-factors based on $F^{2}$ are statistically about twice as large as those based on $F$, and $R$ - factors based on ALL data will be even larger.

Fractional atomic coordinates and isotropic or equivalent isotropic displacement parameters $\left(\AA^{2}\right)$

\begin{tabular}{llllll}
\hline & $x$ & $y$ & $z$ & $U_{\text {iso }} * U_{\text {eq }}$ & Occ. $(<1)$ \\
\hline $\mathrm{Nd} 1$ & $0.24279(2)$ & $0.01102(2)$ & 0.2500 & $0.00756(7)$ & $0.887(5)$ \\
$\mathrm{Ca} 1$ & $0.24279(2)$ & $0.01102(2)$ & 0.2500 & $0.00756(7)$ & $0.113(5)$ \\
$\mathrm{Nd} 2$ & 0.6667 & 0.3333 & $-0.00110(5)$ & $0.00906(10)$ & $0.546(4)$ \\
$\mathrm{Ca} 2$ & 0.6667 & 0.3333 & $-0.00110(5)$ & $0.00906(10)$ & $0.454(4)$ \\
$\mathrm{Si} 1$ & $0.37185(11)$ & $0.40114(11)$ & 0.2500 & $0.0077(2)$ & \\
$\mathrm{O} 1$ & $0.4886(3)$ & $0.3232(3)$ & 0.2500 & $0.0127(5)$ & \\
$\mathrm{O} 2$ & $0.4707(3)$ & $0.5974(3)$ & 0.2500 & $0.0144(5)$ & \\
$\mathrm{O} 3$ & $0.2528(2)$ & $0.3424(3)$ & $0.0659(3)$ & $0.0209(5)$ & \multirow{2}{*}{0.88} \\
$\mathrm{O} 4$ & 0.0000 & 0.0000 & 0.2500 & $0.0141(10)$ & \\
\hline
\end{tabular}

Atomic displacement parameters $\left(\AA^{2}\right)$

\begin{tabular}{lllllll}
\hline & $U^{11}$ & $U^{22}$ & $U^{33}$ & $U^{12}$ & $U^{13}$ & $U^{23}$ \\
\hline $\mathrm{Nd} 1$ & $0.00740(10)$ & $0.00713(10)$ & $0.00733(9)$ & $0.00301(7)$ & 0.000 & 0.000 \\
$\mathrm{Ca} 1$ & $0.00740(10)$ & $0.00713(10)$ & $0.00733(9)$ & $0.00301(7)$ & 0.000 & 0.000 \\
$\mathrm{Nd} 2$ & $0.00913(12)$ & $0.00913(12)$ & $0.00892(15)$ & $0.00456(6)$ & 0.000 & 0.000 \\
$\mathrm{Ca} 2$ & $0.00913(12)$ & $0.00913(12)$ & $0.00892(15)$ & $0.00456(6)$ & 0.000 & 0.000 \\
$\mathrm{Si} 1$ & $0.0069(4)$ & $0.0079(4)$ & $0.0085(4)$ & $0.0039(3)$ & 0.000 & 0.000 \\
O1 & $0.0116(12)$ & $0.0187(13)$ & $0.0123(12)$ & $0.0108(10)$ & 0.000 & 0.000 \\
O2 & $0.0120(12)$ & $0.0085(11)$ & $0.0222(14)$ & $0.0049(9)$ & 0.000 & 0.000 \\
O3 & $0.0158(9)$ & $0.0382(13)$ & $0.0131(9)$ & $0.0166(9)$ & $-0.0042(7)$ & $-0.0098(8)$ \\
O4 & $0.0052(12)$ & $0.0052(12)$ & $0.032(3)$ & $0.0026(6)$ & 0.000 & 0.000 \\
\hline
\end{tabular}

Geometric parameters $\left(\AA,{ }^{\circ}\right)$

\begin{tabular}{llll}
\hline $\mathrm{Nd} 1-\mathrm{Nd} 1^{\mathrm{i}}$ & $3.9284(3)$ & $\mathrm{Si} 1-\mathrm{Nd} 2^{\text {viii }}$ & $3.2527(8)$ \\
$\mathrm{Nd} 1-\mathrm{Nd} 1^{\text {ii }}$ & $3.9284(3)$ & $\mathrm{Si} 1-\mathrm{Nd} 2^{\mathrm{xi}}$ & $3.2527(8)$ \\
$\mathrm{Nd} 1-\mathrm{Nd} 2^{\text {iii }}$ & $4.0666(3)$ & $\mathrm{Si} 1-\mathrm{Ca} 2^{\mathrm{xi}}$ & $3.2527(8)$ \\
$\mathrm{Nd} 1-\mathrm{Si} 1$ & $3.2877(9)$ & $\mathrm{Si} 1-\mathrm{Ca} 2^{\text {viii }}$ & $3.2527(8)$ \\
$\mathrm{Nd} 1-\mathrm{Si} 1^{\mathrm{i}}$ & $3.1738(9)$ & $\mathrm{Si} 1-\mathrm{O} 1$ & $1.621(3)$ \\
$\mathrm{Nd} 1-\mathrm{O} 1$ & $2.721(3)$ & $\mathrm{Si} 1-\mathrm{O} 2$ & $1.623(3)$ \\
$\mathrm{Nd} 1-\mathrm{O} 2^{\text {iv }}$ & $2.463(3)$ & $\mathrm{Si} 1-\mathrm{O} 3^{\mathrm{xii}}$ & $1.629(2)$ \\
$\mathrm{Nd} 1-\mathrm{O} 3^{\mathrm{v}}$ & $2.3909(19)$ & $\mathrm{Si} 1-\mathrm{O} 3$ & $1.629(2)$
\end{tabular}




\begin{tabular}{|c|c|c|c|}
\hline $\mathrm{Nd} 1-\mathrm{O} 3^{\mathrm{vi}}$ & $2.3909(19)$ & $\mathrm{O} 1-\mathrm{Nd} 2^{\mathrm{iii}}$ & $2.4230(17)$ \\
\hline $\mathrm{Nd} 1-\mathrm{O}^{\mathrm{i}}$ & $2.547(2)$ & $\mathrm{O} 1-\mathrm{Ca} 2^{\mathrm{iii}}$ & $2.4230(17)$ \\
\hline $\mathrm{Nd} 1-\mathrm{O} 3^{\mathrm{vii}}$ & $2.547(2)$ & $\mathrm{O} 2-\mathrm{Nd} 1^{\mathrm{iii}}$ & $2.463(3)$ \\
\hline $\mathrm{Nd} 1-\mathrm{O} 4$ & $2.2681(2)$ & $\mathrm{O} 2-\mathrm{Ca} 1^{\mathrm{iii}}$ & $2.463(3)$ \\
\hline $\mathrm{Nd} 2-\mathrm{Si}^{\mathrm{vi}}$ & $3.2527(8)$ & $\mathrm{O} 2-\mathrm{Nd} 2^{\text {viii }}$ & $2.4715(18)$ \\
\hline $\mathrm{Nd} 2-\mathrm{Si} 1^{\text {viii }}$ & $3.2527(8)$ & $\mathrm{O} 2-\mathrm{Nd} 2^{\mathrm{xi}}$ & $2.4715(18)$ \\
\hline $\mathrm{Nd} 2-\mathrm{Si}^{\mathrm{ix}}$ & $3.2527(8)$ & $\mathrm{O} 2-\mathrm{Ca} 2^{\mathrm{xi}}$ & $2.4715(18)$ \\
\hline $\mathrm{Nd} 2-\mathrm{O} 1^{\mathrm{iii}}$ & $2.4231(17)$ & $\mathrm{O} 2-\mathrm{Ca} 2^{\text {viii }}$ & $2.4715(18)$ \\
\hline $\mathrm{Nd} 2-\mathrm{O} 1^{\mathrm{iv}}$ & $2.4231(17)$ & $\mathrm{O} 3-\mathrm{Nd} 1^{\mathrm{ii}}$ & $2.547(2)$ \\
\hline $\mathrm{Nd} 2-\mathrm{O} 1$ & $2.4230(17)$ & $\mathrm{O} 3-\mathrm{Nd} 1^{\text {xiii }}$ & $2.3909(19)$ \\
\hline $\mathrm{Nd} 2-\mathrm{O} 2^{\text {viii }}$ & $2.4715(18)$ & $\mathrm{O} 3-\mathrm{Ca} 1^{\mathrm{xiii}}$ & $2.3909(19)$ \\
\hline $\mathrm{Nd} 2-\mathrm{O} 2^{\mathrm{ix}}$ & $2.4715(18)$ & $\mathrm{O} 3-\mathrm{Ca} 1^{1 \mathrm{ii}}$ & $2.547(2)$ \\
\hline $\mathrm{Nd} 2-\mathrm{O} 2^{\mathrm{vi}}$ & $2.4715(18)$ & $\mathrm{O} 3-\mathrm{Nd} 2^{\text {viii }}$ & $2.830(2)$ \\
\hline $\mathrm{Nd} 2-\mathrm{O}^{\mathrm{x}}$ & $2.830(2)$ & $\mathrm{O} 3-\mathrm{Ca} 2^{\text {viii }}$ & $2.830(2)$ \\
\hline $\mathrm{Nd} 2-\mathrm{O} 3^{\text {viii }}$ & $2.830(2)$ & $\mathrm{O} 4-\mathrm{Nd} 1^{\mathrm{ii}}$ & $2.2681(2)$ \\
\hline $\mathrm{Nd} 2-\mathrm{O}^{\mathrm{vi}}$ & $2.830(2)$ & $\mathrm{O} 4-\mathrm{Nd} 1^{\mathrm{i}}$ & $2.2681(2)$ \\
\hline $\mathrm{Si} 1-\mathrm{Nd} 1^{\mathrm{ii}}$ & $3.1738(9)$ & $\mathrm{O} 4-\mathrm{Ca}^{\mathrm{ii}}$ & $2.2681(2)$ \\
\hline $\mathrm{Si} 1-\mathrm{Ca} 1^{\mathrm{ii}}$ & $3.1738(9)$ & $\mathrm{O} 4-\mathrm{Ca} 1^{\mathrm{i}}$ & $2.2681(2)$ \\
\hline $\mathrm{Nd} 1^{\mathrm{ii}}-\mathrm{Nd} 1-\mathrm{Nd} 1^{\mathrm{i}}$ & 60.0 & $\mathrm{O} 3^{\mathrm{viii}}-\mathrm{Nd} 2-\mathrm{Si} 1^{\mathrm{ix}}$ & $92.88(4)$ \\
\hline $\mathrm{Nd} 1^{\mathrm{ii}}-\mathrm{Nd} 1-\mathrm{Nd} 2^{\mathrm{iii}}$ & $103.981(6)$ & 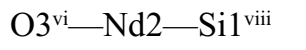 & $92.88(4)$ \\
\hline $\mathrm{Nd} 1^{\mathrm{i}}-\mathrm{Nd} 1-\mathrm{Nd} 2^{\mathrm{iii}}$ & $150.673(5)$ & $\mathrm{O}^{\mathrm{vi}}-\mathrm{Nd} 2-\mathrm{Si}^{\mathrm{vi}}$ & $30.06(4)$ \\
\hline $\mathrm{Si} 1-\mathrm{Nd} 1-\mathrm{Nd} 1^{\mathrm{ii}}$ & $51.249(17)$ & $\mathrm{O} 3^{x}-\mathrm{Nd} 2-\mathrm{Si}^{\mathrm{viii}}$ & $123.63(4)$ \\
\hline 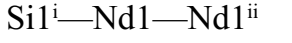 & $113.889(17)$ & $\mathrm{O} 3^{\text {vi }}-\mathrm{Nd} 2-\mathrm{O} 3^{\text {viii }}$ & $117.44(2)$ \\
\hline $\mathrm{Si} 1^{\mathrm{i}}-\mathrm{Nd} 1-\mathrm{Nd} 1^{\mathrm{i}}$ & $53.889(17)$ & $\mathrm{O} 3^{\mathrm{x}}-\mathrm{Nd} 2-\mathrm{O} 3^{\mathrm{vi}}$ & $117.45(2)$ \\
\hline $\mathrm{Si} 1-\mathrm{Nd} 1-\mathrm{Nd} 1^{\mathrm{i}}$ & $111.249(17)$ & $\mathrm{O} 3^{\mathrm{x}}-\mathrm{Nd} 2-\mathrm{O}^{\text {viii }}$ & $117.45(2)$ \\
\hline $\mathrm{Si} 1-\mathrm{Nd} 1-\mathrm{Nd} 2^{\mathrm{iii}}$ & $58.326(14)$ & $\mathrm{Nd} 11^{\mathrm{ii}}-\mathrm{Si} 1-\mathrm{Nd} 1$ & $74.86(2)$ \\
\hline $\mathrm{Si} 1^{\mathrm{i}}-\mathrm{Nd} 1-\mathrm{Nd} 2^{\mathrm{iii}}$ & $134.034(14)$ & $\mathrm{Nd} 1^{\mathrm{ii}}-\mathrm{Si} 1-\mathrm{Nd} 2^{\mathrm{vii}}$ & $81.18(2)$ \\
\hline $\mathrm{Si} 1{ }^{\mathrm{i}}-\mathrm{Nd} 1-\mathrm{Si} 1$ & $165.14(2)$ & $\mathrm{Nd} 1^{\mathrm{ii}}-\mathrm{Si} 1-\mathrm{Nd} 2^{\mathrm{xi}}$ & $81.18(2)$ \\
\hline $\mathrm{O} 1-\mathrm{Nd} 1-\mathrm{Nd} 1^{\mathrm{ii}}$ & $80.66(5)$ & $\mathrm{Nd} 1^{\mathrm{ii}}-\mathrm{Si} 1-\mathrm{Ca} 2^{\mathrm{xi}}$ & $81.18(2)$ \\
\hline $\mathrm{O} 1-\mathrm{Nd} 1-\mathrm{Nd} 1^{\mathrm{i}}$ & $140.66(5)$ & $\mathrm{Nd} 1^{\mathrm{ii}}-\mathrm{Si} 1-\mathrm{Ca} 2^{\mathrm{viii}}$ & $81.18(2)$ \\
\hline $\mathrm{O} 1-\mathrm{Nd} 1-\mathrm{Nd} 2^{\mathrm{iii}}$ & $35.26(3)$ & $\mathrm{Ca} 1 \mathrm{ii}-\mathrm{Si} 1-\mathrm{Nd} 1$ & $74.86(2)$ \\
\hline $\mathrm{O} 1-\mathrm{Nd} 1-\mathrm{Si}^{\mathrm{i}}$ & $165.45(5)$ & $\mathrm{Ca} 1^{\mathrm{ii}}-\mathrm{Si} 1-\mathrm{Nd} 1^{\mathrm{ii}}$ & $0.000(8)$ \\
\hline $\mathrm{O} 1-\mathrm{Nd} 1-\mathrm{Si} 1$ & $29.41(5)$ & $\mathrm{Ca} 1^{\mathrm{ii}}-\mathrm{Si} 1-\mathrm{Nd} 2^{\mathrm{xi}}$ & $81.18(2)$ \\
\hline $\mathrm{O} 2^{\mathrm{iv}}-\mathrm{Nd} 1-\mathrm{Nd} 1^{\mathrm{i}}$ & $120.15(6)$ & $\mathrm{Ca} 1^{\mathrm{ii}}-\mathrm{Si} 1-\mathrm{Nd} 2^{\mathrm{viii}}$ & $81.18(2)$ \\
\hline $\mathrm{O} 2^{\mathrm{iv}}-\mathrm{Nd} 1-\mathrm{Nd} 1^{\mathrm{ii}}$ & $179.85(6)$ & $\mathrm{Ca} 1^{\mathrm{ii}}-\mathrm{Si} 1-\mathrm{Ca} 2^{\mathrm{xi}}$ & $81.18(2)$ \\
\hline $\mathrm{O} 2^{\mathrm{iv}}-\mathrm{Nd} 1-\mathrm{Nd} 2^{\mathrm{iii}}$ & $75.88(5)$ & $\mathrm{Ca} 1^{\mathrm{ii}}-\mathrm{Si} 1-\mathrm{Ca} 2^{\mathrm{viii}}$ & $81.18(2)$ \\
\hline $\mathrm{O} 2^{\mathrm{iv}}-\mathrm{Nd} 1-\mathrm{Si} 1$ & $128.60(6)$ & $\mathrm{Nd} 2^{\mathrm{xi}}-\mathrm{Si} 1-\mathrm{Nd} 1$ & $139.386(19)$ \\
\hline $\mathrm{O} 2^{\mathrm{iv}}-\mathrm{Nd} 1-\mathrm{Si} 1^{\mathrm{i}}$ & $66.27(6)$ & 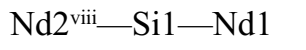 & $139.386(19)$ \\
\hline $\mathrm{O} 2^{\mathrm{iv}}-\mathrm{Nd} 1-\mathrm{O} 1$ & $99.18(8)$ & $\mathrm{Nd} 2^{\mathrm{xi}}-\mathrm{Si} 1-\mathrm{Nd} 2^{\mathrm{viii}}$ & $65.31(2)$ \\
\hline $\mathrm{O} 2^{\mathrm{iv}}-\mathrm{Nd} 1-\mathrm{O}^{\mathrm{i}}$ & $71.01(7)$ & $\mathrm{Nd} 2^{\mathrm{xi}}-\mathrm{Si} 1-\mathrm{Ca} 2^{\mathrm{vii}}$ & $65.31(2)$ \\
\hline $\mathrm{O} 2^{\mathrm{iv}}-\mathrm{Nd} 1-\mathrm{O} 3^{\text {vii }}$ & $71.01(7)$ & $\mathrm{Ca} 2^{\mathrm{xi}}-\mathrm{Si} 1-\mathrm{Nd} 1$ & $139.386(19)$ \\
\hline $\mathrm{O}^{\mathrm{v}}-\mathrm{Nd} 1-\mathrm{Nd} 1^{\mathrm{i}}$ & $110.42(6)$ & $\mathrm{Ca} 2^{\text {viii_- }}$ Si $1-\mathrm{Nd} 1$ & $139.386(19)$ \\
\hline $\mathrm{O} 3^{\mathrm{i}}-\mathrm{Nd} 1-\mathrm{Nd} 1^{\mathrm{i}}$ & $58.32(5)$ & $\mathrm{Ca} 2^{\mathrm{xi}}-\mathrm{Si} 1-\mathrm{Nd} 2^{\mathrm{xi}}$ & $0.000(10)$ \\
\hline $\mathrm{O}^{\mathrm{v}}-\mathrm{Nd} 1-\mathrm{Nd} 1^{\mathrm{ii}}$ & $94.99(5)$ & $\mathrm{Ca} 2^{\text {viii }}-\mathrm{Si} 1-\mathrm{Nd} 2^{\text {viii }}$ & $0.000(10)$ \\
\hline $\mathrm{O} 3^{\mathrm{vi}}-\mathrm{Nd} 1-\mathrm{Nd} 1^{\mathrm{i}}$ & $110.42(6)$ & $\mathrm{Ca} 2^{\mathrm{xi}}-\mathrm{Si} 1-\mathrm{Nd} 2^{\mathrm{vii}}$ & $65.31(2)$ \\
\hline $\mathrm{O} 3^{\mathrm{i}}-\mathrm{Nd} 1-\mathrm{Nd} 1^{\mathrm{ii}}$ & $109.12(5)$ & $\mathrm{Ca} 2^{\mathrm{xi}}-\mathrm{Si} 1-\mathrm{Ca} 2^{\mathrm{viii}}$ & $65.31(2)$ \\
\hline $\mathrm{O} 3^{\mathrm{vii}}-\mathrm{Nd} 1-\mathrm{Nd} 1^{\mathrm{ii}}$ & $109.12(5)$ & $\mathrm{O} 1-\mathrm{Si} 1-\mathrm{Nd} 1$ & $55.53(10)$ \\
\hline
\end{tabular}




$$
\begin{aligned}
& \mathrm{O}^{\mathrm{vii}}-\mathrm{Nd} 1-\mathrm{Nd} 1^{\mathrm{i}} \\
& \mathrm{O}^{\mathrm{vi}}-\mathrm{Nd} 1-\mathrm{Nd} 1^{\mathrm{ii}} \\
& \mathrm{O} 3^{\mathrm{vi}}-\mathrm{Nd} 1-\mathrm{Nd} 2^{\mathrm{iii}} \\
& \mathrm{O} 3^{\mathrm{i}}-\mathrm{Nd} 1-\mathrm{Nd} 2^{\mathrm{iii}} \\
& \mathrm{O} 3^{\mathrm{vii}}-\mathrm{Nd} 1-\mathrm{Nd} 2^{\mathrm{iii}} \\
& \mathrm{O} 3^{\mathrm{v}}-\mathrm{Nd} 1-\mathrm{Nd} 2^{\mathrm{iii}} \\
& \mathrm{O}^{\mathrm{v}}{ }^{-}-\mathrm{Nd} 1-\mathrm{Si} 1 \\
& \mathrm{O}^{2 \mathrm{vi}}-\mathrm{Nd} 1-\mathrm{Si}^{\mathrm{i}} \\
& \text { O3 }{ }^{\mathrm{i}}-\mathrm{Nd} 1-\mathrm{Si}^{\mathrm{i}} \\
& \text { O3 - Nd1-Si1 } \\
& \text { O3 }{ }^{\text {vii }-N d 1-S i 1}{ }^{i} \\
& \mathrm{O}^{\mathrm{v}}-\mathrm{Nd} 1-\mathrm{Si}^{\mathrm{i}}
\end{aligned}
$$

\begin{tabular}{|c|c|c|}
\hline $58.32(5)$ & $\mathrm{O} 1-\mathrm{Si} 1-\mathrm{Nd} 1^{\mathrm{ii}}$ & $130.39(10)$ \\
\hline $94.99(5)$ & $\mathrm{O} 1-\mathrm{Si} 1-\mathrm{Ca} 1^{\mathrm{ii}}$ & $130.39(10)$ \\
\hline $94.52(6)$ & $\mathrm{O} 1-\mathrm{Si} 1-\mathrm{Nd} 2^{\mathrm{xi}}$ & $136.87(6)$ \\
\hline $112.89(5)$ & $\mathrm{O} 1-\mathrm{Si} 1-\mathrm{Nd} 2^{\mathrm{viii}}$ & $136.87(6)$ \\
\hline $146.38(5)$ & $\mathrm{O} 1-\mathrm{Si} 1-\mathrm{Ca} 2^{\text {viii }}$ & $136.87(6)$ \\
\hline $42.90(6)$ & $\mathrm{O} 1-\mathrm{Si} 1-\mathrm{Ca} 2^{\mathrm{xi}}$ & $136.87(6)$ \\
\hline $77.25(5)$ & $\mathrm{O} 1-\mathrm{Si} 1-\mathrm{O} 2$ & $113.16(14)$ \\
\hline $106.70(5)$ & $\mathrm{O} 1-\mathrm{Si} 1-\mathrm{O} 3$ & $111.34(9)$ \\
\hline $30.67(4)$ & $\mathrm{O} 1-\mathrm{Si} 1-\mathrm{O} 3^{\mathrm{xii}}$ & $111.34(9)$ \\
\hline $145.64(5)$ & $\mathrm{O} 2-\mathrm{Si} 1-\mathrm{Nd} 1^{\mathrm{ii}}$ & $116.45(10)$ \\
\hline $30.67(4)$ & $\mathrm{O} 2-\mathrm{Si} 1-\mathrm{Nd} 1$ & $168.69(10)$ \\
\hline $106.70(5)$ & $\mathrm{O} 2-\mathrm{Si} 1-\mathrm{Ca} 1^{\mathrm{ii}}$ & $116.45(10)$ \\
\hline $145.64(5)$ & $\mathrm{O} 2-\mathrm{Si} 1-\mathrm{Nd} 2^{\text {viii }}$ & $47.71(6)$ \\
\hline $77.25(5)$ & $\mathrm{O} 2-\mathrm{Si} 1-\mathrm{Nd} 2^{\mathrm{xi}}$ & $47.70(6)$ \\
\hline $70.49(5)$ & $\mathrm{O} 2-\mathrm{Si} 1-\mathrm{Ca} 2^{\mathrm{xi}}$ & $47.70(6)$ \\
\hline $146.95(5)$ & $\mathrm{O} 2-\mathrm{Si} 1-\mathrm{Ca} 2^{\mathrm{viii}}$ & $47.71(6)$ \\
\hline $70.49(5)$ & $\mathrm{O} 2-\mathrm{Si} 1-\mathrm{O} 3^{\mathrm{xii}}$ & $107.49(10)$ \\
\hline $146.95(5)$ & $\mathrm{O} 2-\mathrm{Si} 1-\mathrm{O} 3$ & $107.49(10)$ \\
\hline $84.96(5)$ & $\mathrm{O}^{\mathrm{xii}}-\mathrm{Si} 1-\mathrm{Nd} 1^{\mathrm{ii}}$ & $52.89(7)$ \\
\hline $84.96(5)$ & $\mathrm{O} 3^{\mathrm{xii}}-\mathrm{Si} 1-\mathrm{Nd} 1$ & $78.94(9)$ \\
\hline $61.28(9)$ & $\mathrm{O} 3-\mathrm{Si} 1-\mathrm{Nd} 1^{\mathrm{ii}}$ & $52.89(7)$ \\
\hline $77.13(4)$ & O3-Si1-Nd1 & $78.94(9)$ \\
\hline $136.63(7)$ & $\mathrm{O} 3-\mathrm{Si} 1-\mathrm{Ca} 1^{\mathrm{ii}}$ & $52.89(7)$ \\
\hline $136.63(7)$ & $\mathrm{O}^{\mathrm{xii}}-\mathrm{Si} 1-\mathrm{Ca} 1^{\mathrm{ii}}$ & $52.89(7)$ \\
\hline $77.13(4)$ & $\mathrm{O} 3^{\mathrm{xii}}-\mathrm{Si} 1-\mathrm{Nd} 2^{\mathrm{xi}}$ & $60.46(9)$ \\
\hline $137.40(11)$ & $\mathrm{O} 3-\mathrm{Si} 1-\mathrm{Nd} 2^{\mathrm{xi}}$ & $111.52(8)$ \\
\hline 30.0 & $\mathrm{O} 3^{\mathrm{xii}}-\mathrm{Si} 1-\mathrm{Nd} 2^{\mathrm{viii}}$ & $111.52(8)$ \\
\hline 30.0 & $\mathrm{O} 3-\mathrm{Si} 1-\mathrm{Nd} 2^{\mathrm{viii}}$ & $60.46(9)$ \\
\hline $130.005(6)$ & $\mathrm{O} 3^{\mathrm{xii}}-\mathrm{Si} 1-\mathrm{Ca} 2^{\mathrm{xi}}$ & $60.46(9)$ \\
\hline $81.249(17)$ & $\mathrm{O} 3^{\mathrm{xii}}-\mathrm{Si} 1-\mathrm{Ca} 2^{\mathrm{viii}}$ & $111.52(8)$ \\
\hline $83.888(17)$ & $\mathrm{O} 3-\mathrm{Si} 1-\mathrm{Ca} 2^{\mathrm{xi}}$ & $111.52(8)$ \\
\hline $110.66(5)$ & $\mathrm{O} 3-\mathrm{Si} 1-\mathrm{Ca} 2^{\mathrm{viii}}$ & $60.46(9)$ \\
\hline $150.15(6)$ & $\mathrm{O} 3^{\mathrm{xii}}-\mathrm{Si} 1-\mathrm{O} 3$ & $105.63(15)$ \\
\hline $104.58(5)$ & $\mathrm{Nd} 22^{\mathrm{iii}}-\mathrm{O} 1-\mathrm{Nd} 1$ & $104.33(7)$ \\
\hline $83.45(5)$ & $\mathrm{Nd} 2-\mathrm{O} 1-\mathrm{Nd} 1$ & $104.33(7)$ \\
\hline $83.45(5)$ & $\mathrm{Nd} 22^{\mathrm{iii}}-\mathrm{O} 1-\mathrm{Nd} 2$ & $93.89(9)$ \\
\hline $104.58(5)$ & $\mathrm{Ca} 2^{\mathrm{iii}}-\mathrm{O} 1-\mathrm{Nd} 1$ & $104.33(7)$ \\
\hline $93.628(14)$ & $\mathrm{Ca} 2^{\mathrm{iii}}-\mathrm{O} 1-\mathrm{Nd} 2$ & $93.89(9)$ \\
\hline $93.628(14)$ & $\mathrm{Ca} 2^{\mathrm{iii}}-\mathrm{O} 1-\mathrm{Nd} 2^{\mathrm{iii}}$ & $0.000(11)$ \\
\hline $93.628(14)$ & $\mathrm{Si} 1-\mathrm{O} 1-\mathrm{Nd} 1$ & $95.06(11)$ \\
\hline $98.07(6)$ & $\mathrm{Si} 1-\mathrm{O} 1-\mathrm{Nd} 2$ & $127.73(7)$ \\
\hline $165.42(5)$ & $\mathrm{Si} 1-\mathrm{O} 1-\mathrm{Nd} 2^{\mathrm{iii}}$ & $127.73(7)$ \\
\hline $94.28(5)$ & $\mathrm{Si} 1-\mathrm{O} 1-\mathrm{Ca} 2^{\mathrm{iii}}$ & $127.73(7)$ \\
\hline $98.07(6)$ & $\mathrm{Nd} 1^{\mathrm{iii}}-\mathrm{O} 2-\mathrm{Nd} 2^{\text {viii }}$ & $115.89(7)$ \\
\hline $98.07(6)$ & $\mathrm{Nd} 1^{\mathrm{iii}}-\mathrm{O} 2-\mathrm{Nd} 2^{\mathrm{xi}}$ & $115.89(7)$ \\
\hline $165.42(5)$ & $\mathrm{Nd} 1^{\mathrm{iii}}-\mathrm{O} 2-\mathrm{Ca} 2^{\text {viii }}$ & $115.89(7)$ \\
\hline $94.28(5)$ & $\mathrm{Nd} 1^{\mathrm{iii}}-\mathrm{O} 2-\mathrm{Ca} 2^{\mathrm{xi}}$ & $115.89(7)$ \\
\hline $165.42(5)$ & $\mathrm{Ca} 1^{1 \mathrm{ii}}-\mathrm{O} 2-\mathrm{Nd} 1^{\mathrm{iii}}$ & $0.000(9)$ \\
\hline
\end{tabular}

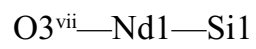

$$
\begin{aligned}
& \mathrm{O}^{\mathrm{vi}} \text { - } \mathrm{Nd} 1-\mathrm{Si} 1 \\
& \mathrm{O}^{\mathrm{v}}-\mathrm{Nd} 1-\mathrm{O} 1 \\
& \mathrm{O} 3{ }^{\mathrm{i}}-\mathrm{Nd} 1-\mathrm{O} 1 \\
& \mathrm{O}^{\mathrm{vi}}-\mathrm{Nd} 1-\mathrm{O} 1
\end{aligned}
$$

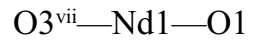

$$
\begin{aligned}
& \mathrm{O}^{\mathrm{vi}}-\mathrm{Nd} 1-\mathrm{O}^{\mathrm{iv}} \\
& \mathrm{O} 3^{\mathrm{v}}-\mathrm{Nd} 1-\mathrm{O} 2^{\mathrm{iv}} \\
& \mathrm{O}^{\mathrm{vii}}-\mathrm{Nd} 1-\mathrm{O}^{\mathrm{i}} \\
& \mathrm{O}^{\text {vi }}-\mathrm{Nd} 1-\mathrm{O}^{\text {vii }} \\
& \mathrm{O}^{2 \mathrm{vi}}-\mathrm{Nd} 1-\mathrm{O}^{\mathrm{i}} \\
& \mathrm{O}^{2}-\mathrm{Nd} 1-\mathrm{O}^{\mathrm{vii}} \\
& \mathrm{O} 3^{\mathrm{v}}-\mathrm{Nd} 1-\mathrm{O}^{\mathrm{i}} \\
& \mathrm{O} 3^{\mathrm{v}}-\mathrm{Nd} 1-\mathrm{O}^{\mathrm{vi}} \\
& \mathrm{O} 4-\mathrm{Nd} 1-\mathrm{Nd} 1^{\mathrm{i}} \\
& \mathrm{O} 4-\mathrm{Nd} 1-\mathrm{Nd} 1^{\mathrm{ii}} \\
& \mathrm{O} 4-\mathrm{Nd} 1-\mathrm{Nd} 2^{\mathrm{iii}} \\
& \text { O4-Nd1-Si1 } \\
& \mathrm{O} 4-\mathrm{Nd} 1-\mathrm{Si}^{1}{ }^{\mathrm{i}} \\
& \mathrm{O} 4-\mathrm{Nd} 1-\mathrm{O} 1 \\
& \mathrm{O} 4-\mathrm{Nd} 1-\mathrm{O}^{\mathrm{iv}} \\
& \mathrm{O} 4-\mathrm{Nd} 1-\mathrm{O}^{\text {vi }} \\
& \mathrm{O} 4-\mathrm{Nd} 1-\mathrm{O}^{\text {vii }} \\
& \mathrm{O} 4-\mathrm{Nd} 1-\mathrm{O}^{\mathrm{i}} \\
& \mathrm{O} 4-\mathrm{Nd} 1-\mathrm{O}^{\mathrm{v}} \\
& \mathrm{Si}^{\mathrm{vi}}-\mathrm{Nd} 2-\mathrm{Si}^{\mathrm{viii}} \\
& \mathrm{Si} 1^{\mathrm{ix}}-\mathrm{Nd} 2-\mathrm{Si} 1^{\text {viii }} \\
& \mathrm{Si}^{\mathrm{ix}}-\mathrm{Nd} 2-\mathrm{Si}^{\mathrm{vi}} \\
& \mathrm{O} 1^{\mathrm{iv}}-\mathrm{Nd} 2-\mathrm{Si}^{1}{ }^{\mathrm{ix}} \\
& \mathrm{O} 1^{\mathrm{iii}}-\mathrm{Nd} 2-\mathrm{Si}^{\mathrm{vi}} \\
& \mathrm{O} 1^{\mathrm{iv}}-\mathrm{Nd} 2-\mathrm{Si}^{1 \mathrm{vi}} \\
& \mathrm{O} 1-\mathrm{Nd} 2-\mathrm{Si}^{\mathrm{vi}} \\
& \mathrm{O} 1^{\mathrm{iii}}-\mathrm{Nd} 2-\mathrm{Si}^{\text {viii }} \\
& \mathrm{O}^{\mathrm{iv}}-\mathrm{Nd} 2-\mathrm{Si}^{\text {viii }}
\end{aligned}
$$

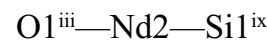

$$
\begin{aligned}
& \mathrm{O} 1-\mathrm{Nd} 2-\mathrm{Si}^{\mathrm{ix}}
\end{aligned}
$$




\begin{tabular}{|c|c|}
\hline $\mathrm{O} 1-\mathrm{Nd} 2-\mathrm{Si}^{\mathrm{viii}}$ & $94.28(5)$ \\
\hline 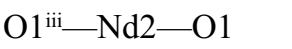 & $72.49(7)$ \\
\hline $\mathrm{O} 1^{\mathrm{iv}}-\mathrm{Nd} 2-\mathrm{O} 1^{\mathrm{iii}}$ & $72.49(7)$ \\
\hline $\mathrm{O} 1^{\mathrm{iv}}-\mathrm{Nd} 2-\mathrm{O} 1$ & $72.49(7)$ \\
\hline $\mathrm{O} 1-\mathrm{Nd} 2-\mathrm{O} 2^{\mathrm{vi}}$ & $125.79(8)$ \\
\hline $\mathrm{O} 1^{\mathrm{iv}}-\mathrm{Nd} 2-\mathrm{O} 2^{\mathrm{vi}}$ & $94.22(6)$ \\
\hline $\mathrm{O} 1-\mathrm{Nd} 2-\mathrm{O} 2^{\mathrm{ix}}$ & $153.93(8)$ \\
\hline $\mathrm{O} 1^{\mathrm{iv}}-\mathrm{Nd} 2-\mathrm{O} 2^{\text {viii }}$ & $153.93(8)$ \\
\hline 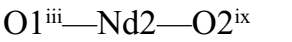 & $94.22(6)$ \\
\hline 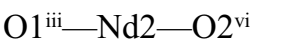 & $153.93(8)$ \\
\hline $\mathrm{O} 1^{\mathrm{iv}}-\mathrm{Nd} 2-\mathrm{O} 2^{\mathrm{ix}}$ & $125.79(8)$ \\
\hline $\mathrm{O} 1-\mathrm{Nd} 2-\mathrm{O} 2^{\text {viii }}$ & $94.22(6)$ \\
\hline $\mathrm{O} 1^{\mathrm{iii}}-\mathrm{Nd} 2-\mathrm{O} 2^{\mathrm{viii}}$ & $125.79(8)$ \\
\hline 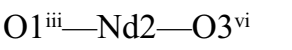 & $139.76(6)$ \\
\hline $\mathrm{O} 1-\mathrm{Nd} 2-\mathrm{O} 3^{\text {viii }}$ & $87.87(7)$ \\
\hline $\mathrm{O} 1^{\mathrm{iv}}-\mathrm{Nd} 2-\mathrm{O} 3^{\mathrm{x}}$ & $68.15(7)$ \\
\hline $\mathrm{O} 1-\mathrm{Nd} 2-\mathrm{O} 3^{\mathrm{vi}}$ & $68.15(7)$ \\
\hline $\mathrm{O} 1^{\mathrm{iii}-\mathrm{Nd} 2-\mathrm{O} 3^{\mathrm{x}}}$ & $87.87(7)$ \\
\hline $\mathrm{O} 1^{\text {iv }}-\mathrm{Nd} 2-\mathrm{O} 3^{\text {viii }}$ & $139.76(6)$ \\
\hline $\mathrm{O} 1^{\mathrm{iv}}-\mathrm{Nd} 2-\mathrm{O} 3^{\mathrm{vi}}$ & $87.87(7)$ \\
\hline $\mathrm{O} 1^{\mathrm{iii}}-\mathrm{Nd} 2-\mathrm{O} 3^{\text {viii }}$ & $68.15(7)$ \\
\hline $\mathrm{O} 1-\mathrm{Nd} 2-\mathrm{O}^{\mathrm{x}}$ & $139.76(6)$ \\
\hline $\mathrm{O} 2^{\mathrm{vi}}-\mathrm{Nd} 2-\mathrm{Si} 1^{\mathrm{ix}}$ & $64.81(6)$ \\
\hline $\mathrm{O} 2^{\mathrm{ix}}-\mathrm{Nd} 2-\mathrm{Si} 1^{\mathrm{vii}}$ & $64.81(6)$ \\
\hline $\mathrm{O} 2^{\mathrm{ix}}-\mathrm{Nd} 2-\mathrm{Si} 1^{\mathrm{vi}}$ & $98.63(5)$ \\
\hline $\mathrm{O} 2^{\mathrm{ix}}-\mathrm{Nd} 2-\mathrm{Si} 1^{\mathrm{ix}}$ & $29.07(6)$ \\
\hline $\mathrm{O} 2^{\text {viii }}-\mathrm{Nd} 2-\mathrm{Si}^{\text {viii }}$ & $29.07(6)$ \\
\hline $\mathrm{O} 2^{\text {viii }}-\mathrm{Nd} 2-\mathrm{Si}^{\mathrm{ix}}$ & $98.63(5)$ \\
\hline 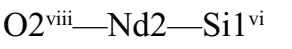 & $64.81(6)$ \\
\hline $\mathrm{O} 2^{\mathrm{vi}}-\mathrm{Nd} 2-\mathrm{Si}^{\mathrm{vi}}$ & $29.07(6)$ \\
\hline $\mathrm{O} 2^{\mathrm{vi}}-\mathrm{Nd} 2-\mathrm{Si}^{\mathrm{viii}}$ & $98.63(5)$ \\
\hline $\mathrm{O} 2^{\mathrm{vi}}-\mathrm{Nd} 2-\mathrm{O} 2^{\text {viii }}$ & $75.14(6)$ \\
\hline 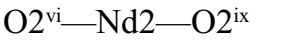 & $75.14(6)$ \\
\hline $\mathrm{O} 2^{\mathrm{ix}}-\mathrm{Nd} 2-\mathrm{O} 2^{\mathrm{viii}}$ & $75.14(6)$ \\
\hline 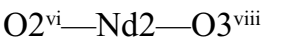 & $125.24(6)$ \\
\hline $\mathrm{O} 2^{\mathrm{vi}-\mathrm{Nd} 2-\mathrm{O} 3^{\mathrm{x}}}$ & $66.19(7)$ \\
\hline $\mathrm{O} 2^{\text {viii }-\mathrm{Nd} 2-\mathrm{O} 3^{\text {viii }}}$ & $58.84(7)$ \\
\hline $\mathrm{O} 2^{\mathrm{ix}}-\mathrm{Nd} 2-\mathrm{O} 3^{\mathrm{x}}$ & $58.84(7)$ \\
\hline $\mathrm{O} 2^{\mathrm{vi}-}-\mathrm{Nd} 2-\mathrm{O} 3^{\mathrm{vi}}$ & $58.85(7)$ \\
\hline $\mathrm{O} 2^{\text {viii-Nd2-OO } 3^{x}}$ & $125.24(6)$ \\
\hline $\mathrm{O} 2^{\mathrm{ix}}-\mathrm{Nd} 2-\mathrm{O} 3^{\text {viii }}$ & $66.19(7)$ \\
\hline $\mathrm{O} 2^{\mathrm{ix}}-\mathrm{Nd} 2-\mathrm{O} 3^{\mathrm{vi}}$ & $125.24(6)$ \\
\hline $\mathrm{O} 2^{\mathrm{viii}}-\mathrm{Nd} 2-\mathrm{O} 3^{\mathrm{vi}}$ & $66.19(7)$ \\
\hline 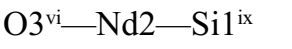 & $123.63(4)$ \\
\hline $\mathrm{O}^{\text {viii }}-\mathrm{Nd} 2-\mathrm{Si}^{\text {viii }}$ & $30.06(4)$ \\
\hline $\mathrm{O}^{\mathrm{x}}-\mathrm{Nd} 2-\mathrm{Si}^{\mathrm{ix}}$ & $30.06(4)$ \\
\hline
\end{tabular}

\begin{tabular}{|c|c|}
\hline 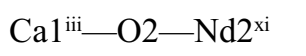 & $115.89(7)$ \\
\hline $\mathrm{Ca} 1^{\mathrm{iii}}-\mathrm{O} 2-\mathrm{Nd} 2^{\text {viii }}$ & $115.89(7)$ \\
\hline $\mathrm{Ca} 1^{\mathrm{iii}}-\mathrm{O} 2-\mathrm{Ca} 2^{\mathrm{xi}}$ & $115.89(7)$ \\
\hline $\mathrm{Ca} 1^{\mathrm{iii}}-\mathrm{O} 2-\mathrm{Ca} 2^{\text {viii }}$ & $115.89(7)$ \\
\hline $\mathrm{Nd} 2^{\mathrm{xi}}-\mathrm{O} 2-\mathrm{Nd} 2^{\text {viii }}$ & $90.49(8)$ \\
\hline $\mathrm{Nd} 2^{\mathrm{xi}}-\mathrm{O} 2-\mathrm{Ca} 2^{\text {viii }}$ & $90.49(8)$ \\
\hline $\mathrm{Nd} 2^{\text {viii }}-\mathrm{O} 2-\mathrm{Ca} 2^{\text {viii }}$ & 0.0 \\
\hline $\mathrm{Ca} 2^{\mathrm{xi}}-\mathrm{O} 2-\mathrm{Nd} 2^{\text {viii }}$ & $90.49(8)$ \\
\hline $\mathrm{Ca} 2^{\mathrm{xi}}-\mathrm{O} 2-\mathrm{Nd} 2^{\mathrm{xi}}$ & 0.0 \\
\hline $\mathrm{Ca} 2^{\mathrm{xi}}-\mathrm{O} 2-\mathrm{Ca} 2^{\text {viii }}$ & $90.49(8)$ \\
\hline $\mathrm{Si} 1-\mathrm{O} 2-\mathrm{Nd} 1^{\mathrm{iii}}$ & $122.72(13)$ \\
\hline $\mathrm{Si} 1-\mathrm{O} 2-\mathrm{Ca} 1^{\mathrm{iii}}$ & $122.72(13)$ \\
\hline $\mathrm{Si} 1-\mathrm{O} 2-\mathrm{Nd} 2^{\mathrm{xi}}$ & $103.23(9)$ \\
\hline $\mathrm{Si} 1-\mathrm{O} 2-\mathrm{Nd} 2^{\mathrm{viii}}$ & $103.23(9)$ \\
\hline $\mathrm{Si} 1-\mathrm{O} 2-\mathrm{Ca} 2^{\mathrm{xi}}$ & $103.23(9)$ \\
\hline $\mathrm{Si} 1-\mathrm{O} 2-\mathrm{Ca} 2^{\text {viii }}$ & $103.23(9)$ \\
\hline $\mathrm{Nd} 1^{\mathrm{xiii}}-\mathrm{O} 3-\mathrm{Nd} 1^{\mathrm{ii}}$ & $116.16(8)$ \\
\hline $\mathrm{Nd} 1^{\mathrm{xiii}}-\mathrm{O} 3-\mathrm{Ca} 1^{\mathrm{ii}}$ & $116.16(8)$ \\
\hline $\mathrm{Nd} 1^{\mathrm{ii}}-\mathrm{O} 3-\mathrm{Nd} 2^{\text {viii }}$ & $101.98(7)$ \\
\hline $\mathrm{Nd} 1^{\text {xiii- }}-\mathrm{O} 3-\mathrm{Nd} 2^{\text {viii }}$ & $101.99(8)$ \\
\hline $\mathrm{Nd} 1^{\mathrm{ii}}-\mathrm{O} 3-\mathrm{Ca} 2^{\mathrm{viii}}$ & $101.98(7)$ \\
\hline $\mathrm{Nd} 1^{\text {xiii }}-\mathrm{O} 3-\mathrm{Ca} 2^{\text {viii }}$ & $101.99(8)$ \\
\hline $\mathrm{Ca} 1^{\mathrm{ii}}-\mathrm{O} 3-\mathrm{Nd} 1^{\mathrm{ii}}$ & $0.000(14)$ \\
\hline $\mathrm{Ca} 1^{\mathrm{xiii}}-\mathrm{O} 3-\mathrm{Nd} 1^{\mathrm{xiii}}$ & 0.0 \\
\hline $\mathrm{Ca} 1^{\mathrm{xiii}}-\mathrm{O} 3-\mathrm{Nd} 1^{\mathrm{ii}}$ & $116.16(8)$ \\
\hline $\mathrm{Ca} 1^{\mathrm{xii}}-\mathrm{O} 3-\mathrm{Ca} 1^{\mathrm{ii}}$ & $116.16(8)$ \\
\hline $\mathrm{Ca} 1^{\mathrm{ii}}-\mathrm{O} 3-\mathrm{Nd} 2^{\text {viii }}$ & $101.98(7)$ \\
\hline 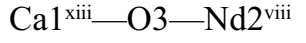 & $101.99(8)$ \\
\hline $\mathrm{Ca} 1^{\mathrm{ii}}-\mathrm{O} 3-\mathrm{Ca} 2^{\text {viii }}$ & $101.98(7)$ \\
\hline $\mathrm{Ca} 1^{\mathrm{xiii}}-\mathrm{O} 3-\mathrm{Ca} 2^{\text {viii }}$ & $101.99(8)$ \\
\hline $\mathrm{Ca} 2^{\text {viii }}-\mathrm{O} 3-\mathrm{Nd} 2^{\text {viii }}$ & $0.000(14)$ \\
\hline $\mathrm{Si} 1-\mathrm{O} 3-\mathrm{Nd} 1^{\mathrm{xiii}}$ & $141.68(11)$ \\
\hline $\mathrm{Si} 1-\mathrm{O} 3-\mathrm{Nd} 1^{\mathrm{ii}}$ & $96.43(9)$ \\
\hline $\mathrm{Si} 1-\mathrm{O} 3-\mathrm{Ca} 1^{\mathrm{xiii}}$ & $141.68(11)$ \\
\hline $\mathrm{Si} 1-\mathrm{O} 3-\mathrm{Ca} 1^{\mathrm{ii}}$ & $96.43(9)$ \\
\hline $\mathrm{Si} 1-\mathrm{O} 3-\mathrm{Nd} 2^{\text {viii }}$ & $89.48(10)$ \\
\hline $\mathrm{Si} 1-\mathrm{O} 3-\mathrm{Ca} 2^{\text {viii }}$ & $89.48(10)$ \\
\hline $\mathrm{Nd} 1^{\mathrm{i}}-\mathrm{O} 4-\mathrm{Nd} 1^{\mathrm{ii}}$ & 120.0 \\
\hline $\mathrm{Nd} 1-\mathrm{O} 4-\mathrm{Nd} 1^{\mathrm{ii}}$ & 120.0 \\
\hline $\mathrm{Nd} 1-\mathrm{O} 4-\mathrm{Nd} 1^{\mathrm{i}}$ & 120.0 \\
\hline $\mathrm{Nd} 1-\mathrm{O} 4-\mathrm{Ca} 1^{\mathrm{ii}}$ & 120.0 \\
\hline $\mathrm{Nd} 1^{\mathrm{i}}-\mathrm{O} 4-\mathrm{Ca} 1^{\mathrm{ii}}$ & 120.0 \\
\hline $\mathrm{Nd} 1-\mathrm{O} 4-\mathrm{Ca}^{\mathrm{i}}$ & 120.0 \\
\hline $\mathrm{Ca} 1^{\mathrm{i}}-\mathrm{O} 4-\mathrm{Nd} 1^{\mathrm{i}}$ & $0.000(16)$ \\
\hline $\mathrm{Ca} 1^{\mathrm{ii}}-\mathrm{O} 4-\mathrm{Nd} 1^{\mathrm{ii}}$ & $0.000(9)$ \\
\hline 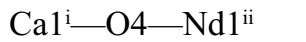 & 120.0 \\
\hline
\end{tabular}


supporting information

$\begin{array}{llll}\mathrm{O}^{2}-\mathrm{Nd} 2-\mathrm{Si}^{\mathrm{xi}} & 92.88(4) & \mathrm{Ca} 1^{\mathrm{i}}-\mathrm{O} 4-\mathrm{Ca}^{\mathrm{ii}} & 120.0 \\ \mathrm{O}^{\mathrm{viii}}-\mathrm{Nd} 2-\mathrm{Si}^{\mathrm{vi}} & 123.63(4) & \end{array}$

Symmetry codes: (i) $-x+y,-x,-z+1 / 2$; (ii) $-y, x-y, z$; (iii) $-x+y+1,-x+1,-z+1 / 2$; (iv) $-y+1, x-y, z$; (v) $y,-x+y, z+1 / 2$; (vi) $y,-x+y,-z$; (vii) $-x+y,-x, z$; (viii) $-x+1,-y+1,-z$; (ix) $x-y+1, x, z-1 / 2$; (x) $x-y+1, x,-z$; (xi) $x-y, x, z+1 / 2$; (xii) $x, y,-z+1 / 2$; (xiii) $x-y, x, z-1 / 2$. 